\title{
Impulsivity and Risk Taking in Bipolar Disorder and Schizophrenia
}

\author{
L Felice Reddy*,', Junghee Lee ${ }^{1,2}$, Michael C Davis ${ }^{1,2}$, Lori Altshuler ${ }^{1,2}$, David C Glahn ${ }^{3,4}$, \\ David J Miklowitz ${ }^{2}$ and Michael F Green ${ }^{1,2}$
}

'VA Greater Los Angeles Healthcare System, MIRECC, Los Angeles, CA, USA; ${ }^{2}$ Semel Institute for Neuroscience and Human Behavior, UCLA, Los Angeles, CA, USA; ${ }^{3}$ Olin Neuropsychiatric Research Center, Institute of Living, Hartford, CT, USA; ${ }^{4}$ Department of Psychiatry, Yale University, New Haven, CT, USA

Impulsive risk taking contributes to deleterious outcomes among clinical populations. Indeed, pathological impulsivity and risk taking are common in patients with serious mental illness, and have severe clinical repercussions including novelty seeking, response disinhibition, aggression, and substance abuse. Thus, the current study seeks to examine self-reported impulsivity (Barratt Impulsivity Scale) and performance-based behavioral risk taking (Balloon Analogue Risk Task) in bipolar disorder and schizophrenia. Participants included 68 individuals with bipolar disorder, 38 with schizophrenia, and 36 healthy controls. Self-reported impulsivity was elevated in the bipolar group compared with schizophrenia patients and healthy controls, who did not differ from each other. On the risk-taking task, schizophrenia patients were significantly more risk averse than the bipolar patients and controls. Aside from the diagnostic group differences, there was a significant effect of antipsychotic (AP) medication within the bipolar group: bipolar patients taking AP medications were more risk averse than those not taking AP medications. This difference in risk taking because of AP medications was not explained by history of psychosis. Similarly, the differences in risk taking between schizophrenia and bipolar disorder were not fully explained by AP effects. Implications for clinical practice and future research are discussed.

Neuropsychopharmacology (2014) 39, 456-463; doi:I0.1038/npp.20 I3.2I8; published online I8 September 20।3

Keywords: schizophrenia; psychopharmacology; behavioral sciences; dopamine

\section{INTRODUCTION}

Impulsiveness is a clinical feature of both schizophrenia and bipolar disorder (Najt et al, 2007; Ouzir, 2013). Broadly, impulsivity refers to a predisposition toward unplanned reactions without consideration of consequences (Moeller et al, 2001) and can include risky decision making, self-reported high-risk attitudes, poor response inhibition, and rapid decision making (Courtney et al, 2012). It is also associated with poor clinical outcomes in patients with bipolar and schizophrenia including substance abuse (Dervaux et al, 2010; Gut-Fayand et al, 2001), suicidal acts (Gut-Fayand et al, 2001), and aggression (Perroud et al, 2011). However, the role that impulsivity has in bipolar disorder and schizophrenia are poorly understood.

Bipolar disorder is often characterized by impulsive behavior and increased tendency to work toward a reward, often without sufficient planning (Johnson et al, 2012). Although risky-impulsive behavior is a diagnostic criterion for mania (American Psychiatric Association, 2000) and

\footnotetext{
*Correspondence: Dr LF Reddy, VA Greater Los Angeles Healthcare System, MIRECC, Building 210, II30I Wilshire Boulevard Room II7, Los Angeles, CA 90073, USA, Tel: +310 478 37IIx42941, Fax: +310 268 4056, E-mail: lena.reddy@va.gov

Received I3 April 2013; revised 23 July 20 I3; accepted 24 July 20 I3; accepted article preview online 21 August 2013
}

bipolar patients consistently show abnormalities on selfreport measures of impulsivity, they do not consistently show deficits on behavioral tasks that require planning and forethought (Holmes et al, 2009; Lombardo et al, 2012). These differences may reflect the multi-faceted nature of impulsivity (Dalley and Roiser, 2012), or the measurement tools used to assess the construct (Ouzir, 2013).

In schizophrenia, there are fewer studies of impulsivity and risk taking. Schizophrenia patients tend to show higher self-reported impulsivity, but the findings on performancebased risk-taking tasks vary, with patients sometimes showing risk aversion or lack of impulsivity (Cheng et al, 2012;) and sometimes an increase in impulsive risk taking (for a review, see Ouzir, 2013). Explanations for nonoptimal risk decisions in schizophrenia include cognitive deficits (Cheng et al, 2012), impaired mental representations of reward (Gold et al, 2012; Heerey et al, 2011), negative symptoms (Heerey et al, 2007), and reward learning abnormalities (Heerey et al, 2008).

Finally, some of the mixed findings in bipolar disorder and schizophrenia regarding impulsivity and risk taking may be attributable to the types of medications patients are taking. The neurochemical basis of impulsivity and risk taking involves dopaminergic, serotonergic, and other neurotransmitter systems (Pattij and Vanderschuren, 2008; Dursun et al, 2000), and these systems are affected by antipsychotic (AP) medications. Some studies find a 
reduction in impulsiveness associated with AP medications (Spivak et al, 1997), whereas others find no such effect in psychotic samples (Ahn et al, 2011; Heerey et al, 2007; Shurman et al, 2005). Thus, it is important to consider the role of AP medications on measures of impulsivity and risk taking.

This is one of the first studies to examine both self-report and behavioral measures of impulsivity in bipolar disorder and schizophrenia. The study had two goals. The primary goal was to conduct a careful analysis of impulsivity using multiple approaches across three groups: bipolar disorder patients, schizophrenia patients, and healthy controls. The secondary goal was to compare subgroups of bipolar disorder patients who differed in terms of AP medications and history of psychosis on measures of impulsivity and risk taking.

\section{METHODS}

\section{Participants}

The total sample $(N=142)$ included 68 participants with bipolar disorder, 38 with schizophrenia, and 36 healthy controls. Patients were recruited from outpatient treatment clinics at the University of California, Los Angeles (UCLA), and the Veterans Affairs Greater Los Angeles Healthcare System (GLA), and from board-and-care residences in Los Angeles. Inclusion criteria for patients included DSM-IV diagnosis of bipolar I, bipolar II, or schizophrenia; diagnoses were confirmed with the Structured Clinical Interview for DSM-IV Axis-I disorders (SCID-I; First et al, 2002). Exclusion criteria for patients included substance dependence in the last 6 months, substance abuse in the last month, and IQ $<70$. Control participants were recruited through internet advertisements and screened with the SCID-I and SCID-II for Axis II disorders (First et al, 1997). Exclusion criteria for control participants included history of schizophrenia, other psychotic disorder, bipolar disorder, recurrent major depressive disorder, substance dependence, or abuse in the past month, history of psychotic or bipolar disorder among first-degree relatives, or any of the following Axis-II disorders: avoidant, paranoid, schizoid, schizotypal, or borderline personality disorder. Exclusion criteria for the entire sample included: history of head injury, identified neurological condition, or lack of English proficiency to understand consent and testing procedures. Patients were considered to be clinically stable, indicated by at least a month since the last mood episode, no medication changes in the previous 6 weeks, no inpatient hospitalization in the previous 3 months, and no changes in housing in the previous 2 months.

Of the 68 patients with bipolar disorder, 46 were diagnosed with bipolar I disorder and 22 with bipolar II disorder. Of the patients with bipolar I, 15 had a history of psychosis and 31 were diagnosed with bipolar I without psychosis. Forty of the bipolar patients were taking AP medications at the time of the study, 28 were not taking AP medications, and 11 were taking lithium or divalproex sodium but not AP medication. Thirty-seven out of thirtynine schizophrenia patients were taking AP medications at the time of testing.

All participants gave written informed consent after receiving a detailed explanation of study procedures, according to procedures approved by the Institutional Review Boards at UCLA and GLA.

\section{Measures}

Clinical ratings. Patients' psychiatric symptoms were evaluated using the expanded 24-item version of the Brief Psychiatric Rating Scale (BPRS; Ventura et al, 1993), the Hamilton Depression Rating Scale (Hamilton, 1960), and the Young Mania Rating Scale (YMRS; Young et al, 1978). All clinical interviewers were trained through the Treatment Unit of the Department of Veterans Affairs VISN 22 Mental Illness Research, Education, and Clinical Center. SCID raters were trained to a minimum kappa of 0.75 and symptom raters were trained to a minimum ICC of 0.80 .

\section{Cognition}

The MATRICS consensus cognitive battery. (MCCB; Nuechterlein and Green, 2006) was used to assess cognitive functioning. The MCCB includes 10 tests to measure seven domains of cognition. The domains include: speed of processing, attention/vigilance, working memory, verbal memory, visual memory, reasoning and problem solving, and social cognition. Standardized T-scores were computed for each of the seven domains, correcting for age and gender. The composite score was based on the average T-score from each of the domains, and served as the primary dependent measure in this study.

\section{Self-reported impulsivity}

The Barratt Impulsiveness Scale. (BIS-11; Patton et al, $1995)$ is a well-validated self-report questionnaire considered to be a reliable indicator of impulsiveness personality traits (Stanford et al, 2009). The measure includes 30 items scored on a 4 -point scale $(1=$ never, $4=$ always $)$ describing common impulsive or non-impulsive behaviors and preferences. Example questions include: 'I say things without thinking;' 'I get easily bored when solving thought problems;' and 'I change jobs.' In addition to the total score, three secondorder factors (attentional, motor, nonplanning) are used in analysis. The dependent measures are summed scores for each of the scales.

\section{Performance-based risk taking}

The Balloon Analogue Risk Task. (BART) is a computerized measure of risk-taking behavior, adapted from an original version by Lejuez et al, 2002. The BART has well-established psychometric properties (White et al, 2008) and predictive validity for real-world risk taking (Cazzell et al, 2012). The task includes a series of 20 balloons displayed on the computer screen. For each balloon, the participant is given the option to earn money by 'pumping' the balloon by pressing a button. Each button press inflates the balloon incrementally and adds $\$ 0.01$ per 'pump' to a bank until a variable point at which the balloon pops. The explosion point of each balloon was between the 1 st and 128th pump, set randomly by the computer. The participant can decide to stop pumping at any time and deposit the accumulated money from that balloon into a permanent bank. If the participant does not stop pumping and the balloon pops, the participant loses all the 
Table I Demographics and Clinical Characteristics of the Sample

\begin{tabular}{|c|c|c|c|c|}
\hline & Bipolar $(N=68)$ & Schizophrenia $(N=38)$ & Controls $(N=36)$ & \\
\hline Age (years) & $44.0(10.6)$ & $44.7(9.2)$ & $41.4(10)$ & $F_{(2,139)}=1.12, P=0.33$ \\
\hline Gender (\% male) & 54.4 & 55.3 & 55.6 & $X^{2}=0.01, P=0.99$ \\
\hline $\begin{array}{l}\text { Race (n) } \\
\text { White }\end{array}$ & 50 & 17 & 16 & \\
\hline Black & 12 & 11 & 11 & \\
\hline Parental education & $14.7(3.1)$ & | $3.7(3.4)$ & I $4.8(3.4)$ & $F_{(2,139)}=1.4, P=0.25$ \\
\hline Age of onset & I $8.3(6.7)$ & $20.2(5.2)$ & - & $F_{(1,102)}=1.91, P=0.17$ \\
\hline BPRS total & $33.4(7.0)$ & $41.3(8.7)$ & - & $F_{(1,102)}=25.3, P<0.01$ \\
\hline BPRS positive symptoms & I.I (0.2) & $2.1(0.9)$ & - & $F_{(1,102)}=96.8, P<0.01$ \\
\hline BPRS negative symptoms & $3.6(1.3)$ & $5.2(2.6)$ & - & $F_{(1,102)}=\mid 4.3, P<0.01$ \\
\hline
\end{tabular}

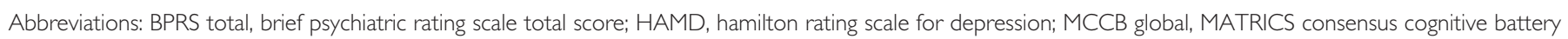
global score; YMRS, young mania rating scale

accumulated money for that trial. The participants were given the actual amount of money they earned during the task, rounded up to the nearest dollar amount. Higher scores are indicative of greater risk-taking propensity (Bornovalova et al, 2005; Lejuez et al, 2002). The three dependent measures are: adjusted pumps (average number of pumps for all unexploded balloons); total amount of money earned; and rate of exploded balloons.

\section{Statistical Analyses}

First, the data were examined for outliers. For the impulsivity and risk-taking variables, none of the patients or controls exceeded three standard deviations above or below the mean for this sample. Second, a series of univariate analyses of variance were conducted to examine group differences on each task. Significant main effects were followed up with paired contrasts using Bonferroni corrections. Third, correlation analyses were used to examine the relation between impulsivity and behavioral risk-taking task. Additional correlation analyses examined the relationships between symptom scales and performance on the impulsivity and risk-taking measures for both patient groups. Finally, $t$-tests were used to examine the extent to which AP medications influenced performance on the measures of impulsivity and risk taking.

\section{RESULTS}

The three groups did not differ in terms of age, gender, and parental education (see Table 1). The two patient groups were equivalent in age of onset of illness. The schizophrenia group had higher symptom scores on the BPRS and the YMRS, and there were no differences between the two patient groups on the HAMD. The three groups differed in terms of cognitive performance on the MCCB, with schizophrenia patients showing the most impairment, bipolar patients intermediate, and healthy controls showing the least impairment.

\section{Group Comparisons on Impulsivity and Risk Taking}

The bipolar group was significantly higher than the schizophrenia patients and the controls on BIS-11 Total scores; the latter two groups did not differ from each other. At the subscale level, the bipolar group performed higher than controls on two impulsivity subscales (attention and motor), but not the third (nonplanning). The schizophrenia group only differed from controls on the attention subscale (see Table 2).

On the BART, there was a significant group effect on the three primary dependent measures (adjusted number of pumps, money earned, and rate exploded) and the $P$-value for each was $<0.01$. The pattern was the same for each of the three primary dependent measures: the schizophrenia group differed significantly from both the bipolar group and controls, who did not differ from each other. In each case, the schizophrenia group showed more risk aversion: they had significantly fewer pumps across balloon trials, earned less money on the task, and had a lower rate of exploded balloons. The groups did not differ in task duration.

Correlations among indices from the two measures were examined within groups (Table 3 ). There are two main findings based on these correlations: looking within each measure, items within the self-reported impulsivity measure (BIS-11) and behavioral measure (BART) were highly intercorrelated to each other for all three groups. Looking between measures, the BIS-11 was not highly related to performance on the BART.

Scores on the impulsivity and risk-taking measures showed only a few relationships with psychiatric symptoms and cognition and none of them were significant after correcting for multiple comparisons. These results are included in Supplementary Table 1.

\section{Subgroup Analyses for Bipolar Disorder}

There were no significant differences between the bipolar I and bipolar II groups on any of the measures of impulsivity or risk taking (Table 4). These two subgroups were 
Table 2 ANOVAs for Impulsivity and Risk Taking

\begin{tabular}{|c|c|c|c|c|c|}
\hline & Bipolar & Schizophrenia & Controls & & Group comparisons \\
\hline \multicolumn{6}{|l|}{$B / S$} \\
\hline Total & $52.3(11)$ & $46.1(12.4)$ & $42.9(8.8)$ & $F_{(2,137)}=9.3, P<0.01$ & $\mathrm{BD}>\mathrm{SCZ}=\mathrm{HC}$ \\
\hline Attention & $12.9(4.6)$ & $11.7(4.7)$ & $8.12(3.4)$ & $F_{(2,137)}=\mid 3.5, P<0.01$ & $\mathrm{BD}=\mathrm{SCZ}>\mathrm{HC}$ \\
\hline Motor & I6.5 (5.2) & | $3.5(5.8)$ & II.4 (4) & $F_{(2,137)}=\mid 1.7, P<0.01$ & $\mathrm{BD}>\mathrm{SCZ}=\mathrm{HC}$ \\
\hline Nonplanning & $22.9(5.5)$ & $20.9(6.4)$ & $23.4(4)$ & $F_{(2,137)}=2.3, P=0.10$ & $\mathrm{BD}=\mathrm{SCZ}=\mathrm{HC}$ \\
\hline \multicolumn{6}{|l|}{ BART } \\
\hline Total adjusted pumps & $35.4(14.7)$ & $21.4(14.2)$ & $38.4(18.7)$ & $F_{(2,137)}=|3.4, P<0.0|$ & $\mathrm{SCZ}<\mathrm{BD}=\mathrm{HC}$ \\
\hline Money earned ${ }^{\mathrm{a}}$ & $451(121)$ & $319(157)$ & $45 \mid(129)$ & $F_{(2,137)}=|3.6, P<0.0|$ & $\mathrm{SCZ}<\mathrm{BD}=\mathrm{HC}$ \\
\hline Rate exploded (\%) & 31 & 17 & 34 & $F_{(2,137)}=|4.3, P<0.0|$ & $\mathrm{SCZ}<\mathrm{BD}=\mathrm{HC}$ \\
\hline Duration in seconds & $389(164)$ & $335(167)$ & $339(164)$ & $F_{(2,137)}=1.7, P=0.18$ & $\mathrm{SCZ}=\mathrm{BD}=\mathrm{HC}$ \\
\hline
\end{tabular}

a Money earned is measured in cents $(\$ 0.0 \mathrm{I})$

Table 3 Correlations among Key Variables within Group

\begin{tabular}{|c|c|c|c|c|c|c|}
\hline & $\mathbf{I}$ & 2 & 3 & 4 & 5 & 6 \\
\hline \multicolumn{7}{|l|}{ Bipolar } \\
\hline \multicolumn{7}{|l|}{ I.BART_adjusted pumps } \\
\hline 2.BART—total \$; & $0.91 *$ & & & & & \\
\hline 3.BART_rate exploded & $0.93 *$ & $0.75 *$ & & & & \\
\hline 4.BIS - attention & $0.25 * *$ & $0.28 * *$ & 0.23 & & & \\
\hline 5.BIS - motor & 0.06 & 0.13 & 0.03 & $0.46 *$ & & \\
\hline 6.BIS_-nonplanning & 0.12 & 0.11 & 0.09 & 0.25 *** & 0.14 & \\
\hline 7.BIS - total & 0.19 & 0.23 & 0.15 & $0.76 *$ & $0.73 *$ & $0.67 *$ \\
\hline
\end{tabular}

\section{Schizophrenia}

I.BART—adjusted pumps

2.BART-total \$;

3.BART — rate exploded

4.BIS - attention

5.BIS - motor

6.BIS - nonplanning

7.BIS-total

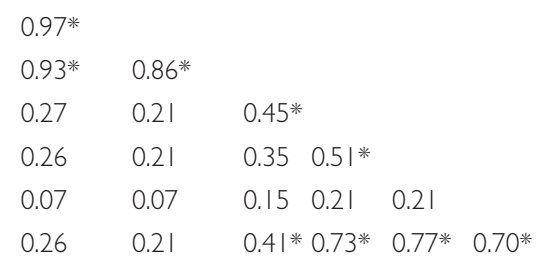

Controls

\begin{tabular}{|c|c|c|c|c|c|}
\hline 2.BART—total \$; & $0.73 * *$ & & & & \\
\hline 3.BART_rate exploded & $0.95 *$ & $0.59 * *$ & & & \\
\hline BIS - attention & -0.11 & -0.04 & -0.07 & & \\
\hline BIS-motor & 0.20 & 0.03 & 0.28 & $0.58 *$ & \\
\hline BS-nonplanning & -0.04 & -0.17 & -0.06 & 0.28 & $0.37 * *$ \\
\hline S-total & 0.03 & -0.08 & -0.07 & $0.77 *$ & $0.84 * 0.73$ \\
\hline
\end{tabular}

Abbreviations: BART, average pumps, adjusted average pumps;

BIS, attention, BIS-I I Attentional impulsivity; BIS - motor, BIS-I I motor impulsivity; BIS, nonplanning, BIS-I I nonplanning impulsivity.

*** $P<0.01$.

$* P<0.05$

comparable and did not differ significantly on any of the demographic variables or on cognitive abilities and clinical symptoms.
Notably, bipolar patients taking AP medications $(n=40)$ had significantly lower scores on the BIS-11 and also the BART (fewer pumps, less money earned, lower rate exploded) than bipolar patients not taking AP meds $(n=28)$. We further explored this effect of AP medications on risk taking and impulsivity in two ways. First, we compared the bipolar patients taking, to those not taking, AP medications on clinical symptoms. We found no differences in bipolar patients who were and were not taking AP medications in symptom severity on any of the symptom measures (BPRS total, BPRS positive, BPRS negative, YMRS, HAMD; Supplementary Table 2). Second, to determine whether the effects of AP medications were explained by history of psychosis, we examined the effects of AP medications only in the 51 bipolar patients who did not have a history of psychosis (we had fewer patients with a history of psychosis). The AP effects were significant (see the last two columns in Table 4) such that patients taking AP medications had significantly lower scores on the BIS and the BART.

Our results indicate not only a diagnostic difference between bipolar disorder and schizophrenia, but also a difference within bipolar disorder based on the use of AP medications. Hence, we wanted to determine whether the AP effects explain the diagnostic group effects on self-report and behavioral impulsivity. We did this by comparing the schizophrenia group to only those bipolar patients who were taking AP medications. The two groups differed significantly on the BART in that the bipolar subgroup showed significantly more risk taking than the schizophrenia group. There were no significant differences between the bipolar subgroup and schizophrenia group on the BIS total or attention or nonplanning subscales (Table 5).

To evaluate whether the group differences between schizophrenia patients and bipolar patients taking AP medications could be due to differences in dosing, we converted the AP medication doses to $\mathrm{CPZ}$ equivalents. The two groups did not differ significantly in CPZ dosage (Table 5). To cross-check the effect of dosing on the analyses, we repeated the comparison between schizophrenia and bipolar patients taking AP medications with $\mathrm{CPZ}$ equivalents as a covariate in ANCOVAs and the results were essentially unchanged (that is, all comparisons 
Table 4 Bipolar Disorder Subgroup Analyses

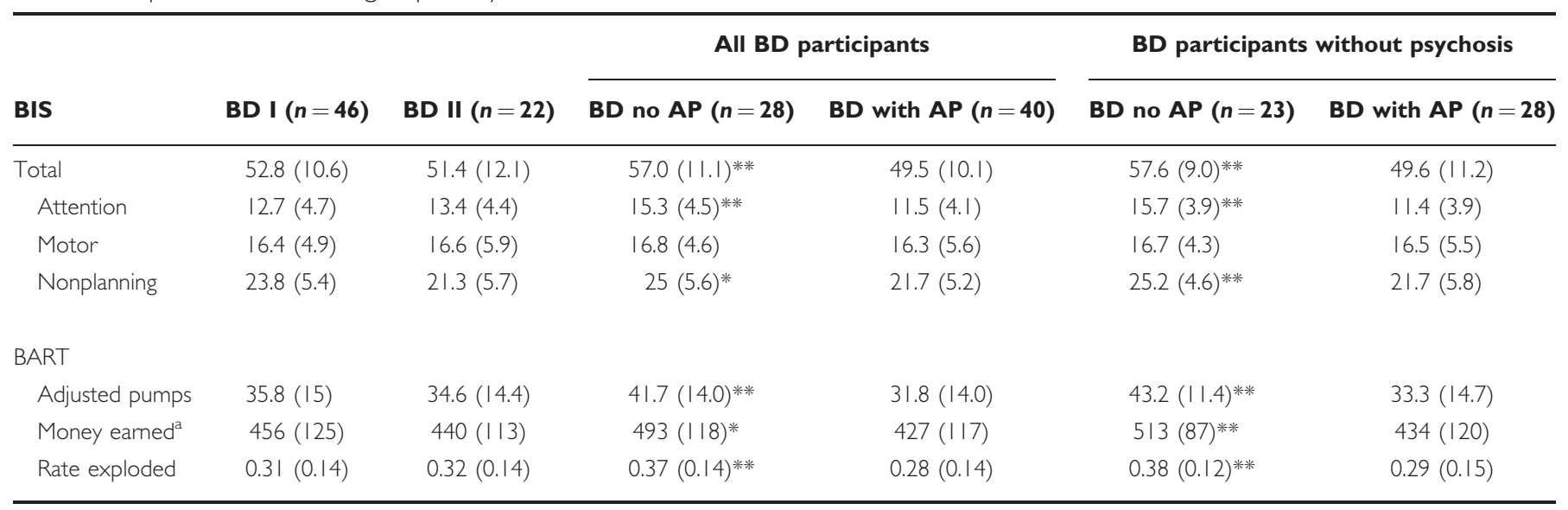

** $P<0.0$ I. $* P<0.05$

a Money earned is measured in cents $(\$ 0.01)$.

Table 5 Bipolar Subgroup and Schizophrenia Group Comparisons

\begin{tabular}{|c|c|c|c|}
\hline \multicolumn{4}{|c|}{ BD with AP Schizophrenia } \\
\hline \multicolumn{4}{|l|}{ BIS } \\
\hline Total & $49.5(10.2)$ & $46.1(12.4)$ & $F(1,78)=1.81, P=N S$ \\
\hline Attention & I I.3 (4.0) & I I.7 (4.7) & $F(1,78)=0.16, P=N S$ \\
\hline Motor* & I6.4 (5.6) & | 3.5 (5.8) & $F(I, 78)=4.84, P=0.03$ \\
\hline Nonplanning & $21.9(5.5)$ & $20.9(6.4)$ & $\mathrm{F}(\mathrm{I}, 78)=0.53, P=\mathrm{NS}$ \\
\hline \multicolumn{4}{|l|}{ BART } \\
\hline Adjusted pumps*** & $32.5(14.7)$ & $21.4(14.2)$ & $F(1,78)=|| .64, P<0.0 \mid$ \\
\hline Money earned ${ }^{a * * *}$ & $429(120)$ & $319(157)$ & $F(1,78)=|2.21, P<0.0|$ \\
\hline Rate exploded* & $0.28(0.14)$ & $0.17(0.14)$ & $F(1,78)=|2.08, P<0.0|$ \\
\hline CPZ equivalents & $243.6(191)$ & $323.0(236)$ & $F(1,57)=1.99, P=N S$ \\
\hline
\end{tabular}

that are significant in Table 5 remain significant with the covariate).

\section{DISCUSSION}

In this study, we evaluated impulsivity and risk taking in schizophrenia and bipolar disorder, assessed with both selfreport and performance-based measures. Several key conclusions emerge from these analyses. First, there were large differences on measures of risk-taking behavior between the two clinical disorders: bipolar patients scored significantly higher than controls on self-reported impulsivity, but performed comparably to controls on behavioral risk taking. In contrast, the schizophrenia patients were comparable to controls on self-reported impulsivity, but showed significantly more behavioral risk aversion than healthy controls or bipolar patients. These findings are consistent with previous studies of bipolar disorder, and largely consistent with previous studies of schizophrenia. Specifically, bipolar patients self-report increased impulsivity, even out of manic episodes (Peluso et al, 2007; Najt et al,
2007; Lombardo et al, 2012). Furthermore, behavioral performance on measures of impulsivity varies in euthymic BP patients: one study found increased impulsivity only in bipolar patients with co-occurring alcohol use disorders (Holmes et al, 2009), and another found behavioral task impulsivity not to be elevated in euthymic patients (Swann et al, 2003). In addition, performance on the BART was not found to correlate with severity of mood symptoms (Holmes et al, 2009).

The previous findings with schizophrenia are more mixed. Schizophrenia patients have reported higher levels of impulsivity on the BIS-11 than controls (Nolan et al, 2011), which is in the direction we found, although it was not significant in our sample. Our findings are consistent with a previous study of the BART that showed significantly less risk taking in schizophrenia patients than controls (Cheng et al, 2012). Some studies observed an apparent increase in impulsivity in schizophrenia on different types of tasks, such as stop-signal and delay discounting (Enticott et al, 2008; Heerey et al, 2007, 2011). However, impulsivity is multi-faceted and these tasks differ from the BART in that they involve both motor impulsivity and cognitive inhibition. Consistent with previous studies, we found that self-reported impulsivity and behavioral risk taking do not correlate highly with each other (Enticott et al, 2008; Ouzir, 2013).

Our study is the first to clearly detect an AP medication effect on risk taking in bipolar disorder patients. These AP effects were not explained by a history of psychosis in the bipolar patients, and the AP effects did not fully account for the diagnostic differences in impulsivity and risk taking. Past studies have hypothesized medication effects may explain changes in performance on measures of impulsiveness but underpowered samples may have prevented detection of such an effect.

Our findings are largely consistent with the characteristic clinical features of the two diagnostic groups. The main group difference between the bipolar patients and controls was self-reported impulsivity (BIS-11), whereas the main group difference between the schizophrenia patients and controls was behavioral risk taking (BART). It may be that self-reported measures are indicative of long-standing 
self-perceptions. The BIS-11 asks the respondent to indicate the degree to which stereotypically impulsive behaviors are characteristic of him or her. If the bipolar patient has salient memories of impulsive actions, or identifies with the behavioral descriptions, they may be inclined to overreport impulsive traits. The difference we found in selfreported impulsiveness between bipolar patients on and off AP medications is consistent with recent studies in other disorders that have found all types of impulsivity (ie, behavioral, nonplanning, and cognitive) are partially state related (Corruble et al, 2003). The BIS-11 may be sensitive to these state-relevant fluctuations. In contrast to the bipolar patients as a whole, the schizophrenia patients were particularly risk averse, perhaps due to repeated exposures to failure and unsuccessful attempts at challenging or new tasks. This is consistent with the current conceptualization of defeatist beliefs as predictive of functional outcome in schizophrenia (Beck and Rector, 2005). Repeated discouraging experiences throughout the development and adulthood eventually cultivate negative self-views and defeatist beliefs, which can lead to loss of motivation in people with schizophrenia (Green et al, 2012). Hence, in bipolar disorder there may be a tendency for people to selfperceive, and report, impulsivity as a pervasive characteristic, which may lead to overly inflated scores on the BIS- 11 . In schizophrenia, defeatist beliefs may lead to inhibited performance and risk aversion on the BART.

There could also be differences in the underlying neurobiology of self-reflection contributing to the diagnostic differences in self-report and performance. Although the neural systems involved in self-reflection are not as well understood as those underlying risk taking, there appears to be a network of brain regions associated with self-reflective processing. For example, patients with schizophrenia show decreased activation in the posterior cingulate cortex and precuneus during a self-reflection task compared with healthy controls (van der Meer et al, 2012). This decreased ability to couple memories of past experiences with sense of self could lead to the discrepancy between normal levels of self-reported impulsivity and the decreased risktaking behaviors in this group. It is possible that bipolar disorder and/or AP medications could affect these neural systems and thereby affect self-reflection on impulsivity, although this has not yet been demonstrated. Overall, it appears that differences on the two measures between patient groups are owing to the fact that the BIS-11 relies on self-perception, whereas the BART relies on behavioral performance. This is consistent with previous findings that self-reported behavioral styles have low associations with measures of specific behaviors in the laboratory (Aichert et al, 2012).

The finding of an AP effect on impulsivity and risk taking may help to explain previous mixed results from studies of both schizophrenia and bipolar disorder (ie, decreased or increased impulsive decision-making). These inconsistencies in past research (eg, Holmes et al, 2009; Lombardo et al, 2012) might be explained if previous studies had different proportions of patients on and off AP medications, or were limited in testing these effects because of small sample sizes.

In clinical practice, it is a commonplace for AP medications to be prescribed in addition to anticonvulsant mood stabilizers, or in place of anticonvulsants, for patients with bipolar disorder. Our findings are consistent with clinical impressions that AP medications may reduce impulsivity and risk-taking behavior. Although there are certain risks associated with AP medications (movement symptoms, metabolic side effects, etc.), this potential effect on impulsivity could be an added benefit for certain patients.

Our participants who were taking AP medications were primarily taking second-generation APs. These agents have varied affinities for numerous neurotransmitter receptors, including those for serotonin and dopamine, that have been associated with risk taking and impulsivity (Dalley and Roiser, 2012; Pattij and Vanderschuren, 2008). A common mechanism across these agents is antagonism of dopamine D2 receptors (Janicak et al, 2011). However, the specific role of dopamine signaling on impulsivity and risk taking is complex. For example, enhancing dopaminergic signaling in Parkinson's Disease with the D2/D3 agonist pramipexole has been shown to increase risk taking behavior on a lottery task, and this is mirrored by the increased rate of impulse control disorders found in Parkinson's Disease patients treated with dopaminergic agonists (Riba et al, 2008). In contrast, psychostimulants that enhance dopaminergic signaling (eg, amphetamine or methylphenidate) decrease risk-taking behaviors in frontotemporal dementia and ADHD (Dalley and Roiser, 2012; Rahman et al, 2005).

Animal studies further illustrate the complexity of dopaminergic involvement in risk-taking behavior. The complexity is owing to the multiple brain regions governing these behaviors, including the basal ganglia and several regions of the prefrontal cortex (Kim and Lee, 2011). Impairments in these regions (eg, the orbitofrontal cortex in frontotemporal dementia) can lead to different neural mechanisms for increased impulsivity, as well as differential responses to dopaminergic medications. In the rat, D2 stimulation increased and D2 antagonism decreased risk taking (St Onge and Floresco, 2009). The results were attributed to the role of the nucleus accumbens, as part of the mesolimbic pathway (St Onge and Floresco, 2009). In contrast, opposite results were found in a different rat model of risk-taking behavior (which included footshock punishment), with D2 receptor activation decreasing risktaking behavior and D2 antagonism attenuating this effect (Simon et al, 2011). This effect was attributed to pharmacological effects of dopaminergic receptors located in both prefrontal and striatal brain regions. The authors concluded that the differences in results between the two studies could be explained by the risk of footshock punishment having greater motivational salience than the alternative reward (Simon et al, 2011). The BART more closely resembled the task in St Onge and Floresco (2009) in that there was no active punishment but instead merely the loss of reward.

It is possible that AP medications change symptoms, which in turn, change risk taking. This explanation is plausible, but our data are not fully consistent with it. We considered this possibility in two ways. First, we examined the correlations between clinical symptoms and risk taking within both groups and did not find any significant correlations after correction (Supplementary Table 1). Second, we compared the bipolar group with and without AP meds on their clinical symptoms and found no differences (Supplementary Table 2). It is possible that AP medications work through symptoms, but not ones assessed 
in this study. For example, some types of negative symptoms that are not assessed with the scales in this study might be more closely related to behavioral impulsiveness.

Alternatively, AP medications might exert their effects on risk taking directly and not through positive, negative, or manic symptoms (at least as defined by the YMRS). One small study of AP naïve first-episode schizophrenia patients suggests that the paracingulate cortex, an area considered critical for performance monitoring, may become hypoactive with AP medications (Yücel et al, 2007). Animal models consistently show that pharmacological agents affecting DA and serotonin systems alter impulsive behavior- and reward-related decision-making (Dalley and Roiser, 2012).

The current study has some strengths and several limitations that suggest avenues for further study. The combined use of both self-report and behavioral measures allowed us to more fully characterize the complexity of impulsivity and risk taking in two clinical samples. The tendency to treat a large subgroup of bipolar patients with AP medications for mood stabilization (even without a history of psychosis) essentially provided a naturalistic behavioral psychopharmacology manipulation. Given our sample size of bipolar patients, we were able to capitalize on this treatment trend for AP medications to conduct informative comparisons.

Limitations of the current study include the cross-sectional design and absence of random assignment to medication status. Extending the current findings by including bipolar patients in manic, depressed, and euthymic episodes may further elucidate the role AP medications have in predicting impulsivity and risk taking. Although we controlled for symptom severity, bipolar type, and history of psychosis in our analyses, we cannot rule out the possibility that the bipolar patients on and off of AP medications were qualitatively different befofre AP treatment. In addition, findings from other research groups suggest that deficits in cognition, motivation, or reward salience may lead to impaired performance among schizophrenia subjects on the BART. The current study, however, was not able to disentangle these factors. Future studies should include systematic manipulations of attentional demands, reward learning contingencies, or motivational attributes.

\section{FUNDING AND DISCLOSURE}

Dr Green has served as a consultant for Abbott Laboratories, Amgen, Biogen, Mnemosyne, Roche, and Shire. He has received research support from Amgen. Dr Altshuler has received past funding from Sepracor (advisory board honoraria, January 2010), Eli Lilly (consultant, September 2010), and Takeda Pharmaceuticals North America, and H. Lundbeck A/S (advisory board honoraria, October 2012); and past and potential future funding from Sunovion Pharmaceuticals Inc. (advisory board honoraria, Jan 2013). Dr Miklowitz has received research funding from the National Institute of Mental Health (NIMH), the National Association for Research on Schizophrenia and Depression (NARSAD), the Danny Alberts Foundation, the Attias Family Foundation, and the Robert L Sutherland Founda- tion; and book royalties from Guilford Press and John Wiley \& Sons. Dr Davis is named as a co-inventor on a University of California provisional patent application entitled 'Methods of using (S)-Hydroxyzine and (R)-Hydroxyzine.' Dr Reddy, Dr Glahn, and Dr Lee have no conflicts of interests or disclosures.

\section{REFERENCES}

Ahn W, Rass O, Fridberg DJ, Bisharz AJ, Forsyth JK, Breier A et al (2011). Temporal discounting of rewards in patients with bipolar disorder and schizophrenia. J Abnorm Psychol 120: 911-921.

Aichert DS, Wöstmann NM, Costa A, Macare C, Wenig JR, Möller HJ et al (2012). Associations between trait impulsivity and prepotent response inhibition. J Clin Exp Neuropsychol 34: 1016-1032.

American Psychiatric Association (2000). Diagnostic and Statistical Manual of Mental Disorders, 4th edition, Text Revision (DSM-IVTR). American Psychiatric Press: Washington, DC.

Beck AT, Rector NA (2005). Cognitive approaches to schizophrenia: theory and therapy. Annu Rev Clin Psychol 1: 577-606.

Bornovalova M, Daughters SB, Richards J, Hernandez GD, Lejuez CW (2005). Differences in impulsivity and risk taking propensity between urban crack/cocaine and heroin users. Exp Clin Psychopharmacol 13: 311-318.

Cazzell M, Li L, Lin Z, Patel SJ, Liu H (2012). Comparison of neural correlates of risk decision making between genders: an exploratory FNIRS study of the Balloon Analogue Risk Task (BART). Neuroimage 62: 1896-1911.

Cheng GF, Tang JY, Li FS, Lau EY, Lee TC (2012). Schizophrenia and risk-taking: impaired reward but preserved punishment processing. Schizophr Res 136: 122-127.

Corruble EA, Benyamina A, Bayle F, Falissard B, Hardy P (2003). Understanding impulsivity in severe depression? A psychometrical contribution. Prog Neuropsychopharmacol Biol Psychiatry 27: 829-833.

Courtney KE, Arellano R, Barkley-Levenson E, Gálvan A, Poldrack RA, Mackillop J et al (2012). The relationship between measures of impulsivity and alcohol misuse: An integrative structural equation modeling approach. Alcohol Clin Exp Res 36: 923-931.

Dalley JW, Roiser JP (2012). Dopamine, serotonin and impulsivity. Neuroscience 215: 42-58.

Dervaux A, Goldberger C, Gourion D, Bourdel M, Laqueille X, Lôo $\mathrm{H}$ et al (2010). Impulsivity and sensation seeking in cannabis abusing patients with schizophrenia. Schizophr Res 123: 278-280.

Dursun SM, Szemis A, Andrews H, Whitaker P, Reveley MA (2000). Effects of clozapine and typical antipsychotic drugs on plasma 5-HT turnover and impulsivity in patients with schizophrenia. J Psychiatry Neurosci 25: 347-352.

Enticott PG, Ogloff JRP, Bradshaw JL (2008). Response inhibition and impulsivity in schizophrenia. Psychiatry Res 157: 251-254.

First MB, Spitzer RL, Gibbon M, Williams JBW (2002). Structured Clinical Interview for DSM-IV-TR Axis I Disorders, Research Version, Patient Edition. (SCID-I/P). Biometrics Research, New York State Psychiatric Institute: New York.

First MB, Gibbon M, Spitzer RL, JBW Williams, Benjamin LS (1997). Structured Clinical Interview for DSM-IV Axis II Personality Disorders, (SCID-II). American Psychiatric Press: Washington, DC.

Gold JM, Waltz JA, Matveeva TM, Kasanova Z, Strauss GP, Herbener ES et al (2012). Negative symptoms and the failure to represent the expected reward value of actions. Arch Gen Psychiatry 69: 129-138.

Green MF, Hellemann G, Horan WP, Lee J, Wynn JK (2012). From perception to functional outcome in schizophrenia. Arch Gen Psychiatry 69: 1216-1224.

Gut-Fayand A, Dervaux A, Olié JP, Lôo H, Poirier MF, Krebs MO (2001). Substance abuse and suicidality in schizophrenia: a 
common risk factor linked to impulsivity. Psychiatry Res 102: $65-72$.

Hamilton M (1960). A rating scale for depression. J Neurol Neurosurg Psychiatry 23: 56-62.

Heerey EA, Bell-Warren KR, Gold JM (2008). Decision-making impairments in the context of intact reward sensitivity in schizophrenia. Biol Psychiatry 64: 62-69.

Heerey EA, Matveeva TM, Gold JM (2011). Imagining the future: degraded representations of future rewards and events in schizophrenia. J Abnorm Psychol 120: 483-489.

Heerey EA, Robinson BM, McMahon RP, Gold JM (2007). Delay discounting in schizophrenia. Cogn Neuropsychiatry 12: 213-221.

Holmes M, Bearden CE, Barguil M, Fonseca M, Monkul E, Nery FG et al (2009). Conceptualizing impulsivity and risk taking in bipolar disorder: importance of history of alcohol abuse. Bipolar Disorders 11: 33-40.

Janicak PG, Marder SR, Pavuluri MN (2011). Principles and Practice of Psychopharmacotherapy, 5th edn. Harvey Whitney Books: Philadelphia, PA.

Johnson SL, Edge MD, Holmes M, Carver CS (2012). The behavioral activation system and mania. Annu Rev Clin Psychol 8: 243-267.

Kim S, Lee D (2011). Prefrontal cortex and impulsive decision making. Biol Psychiatry 69: 1140-1146.

Lejuez CW, Read JP, Kahler CW, Richards JB, Ramsey SE, Stuart GL (2002). Evaluation of a behavioral measure of risk taking: the Balloon Analogue Risk Task (BART). J Exp Psychol Appl 8: 75-84.

Lombardo LE, Bearden CE, Barrett J, Brumbaugh MS, Pittman B, Frangou S (2012). Trait impulsivity as an endophenotype for bipolar I disorder. Bipolar Disorders 14: 565-570.

Moeller FG, Barratt ES, Dougherty DM, Schmitz JM, Swann AC (2001). Psychiatric aspects of impulsivity. Am J Psychiatry 158: 1783-1793.

Najt P, Perez J, Sanches M, Peluso M, Glahn D, Soares J (2007). Impulsivity and bipolar disorder. Eur Neuropsychopharmacol 17: 313-320.

Nolan KA, D'Angelo D, Hoptman MJ (2011). Self-report and laboratory measures of impulsivity in patients with schizophrenia or schizoaffective disorder and healthy controls. Psychiatry Res 187: 301-303.

Nuechterlein KH, Green MF (2006). MATRICS Consensus Cognitive Battery. MATRICS Assessment: Los Angeles, CA.

Ouzir M (2013). Impulsivity in schizophrenia: A comprehensive update. Aggress Viol Behav 18: 247-254.

Pattij T, Vanderschuren LJ (2008). The neuropharmacology of impulsive behaviour. Trends Pharmacol Sci 29: 192-199.

Patton JH, Stanford MS, Barratt ES (1995). Factor structure of the Barratt Impulsiveness Scale. J Clin Psychol 51: 768-774.
Peluso MM, Hatch JP, Glahn DC, Monkul ES, Sanches MM, Najt PP (2007). Trait impulsivity in patients with mood disorders. J Affect Disord 100: 227-231.

Perroud N, Baud P, Mouthon D, Courtet P, Malafosse A (2011). Impulsivity, aggression and suicidal behavior in unipolar and bipolar disorders. J Affect Disord 134: 112-118.

Rahman S, Robbins TW, Hodges JR, Mehta MA, Nestor PJ, Clark L et al (2005). Methylphenidate ('Ritalin') can ameliorate abnormal risk-taking behavior in the frontal variant of frontotemporal dementia. Neuropsychopharmacol 31: 651-658.

Riba J, Krämer UM, Heldmann M, Richter S, Münte TF (2008). Dopamine agonist increases risk taking but blunts rewardrelated brain activity. PLoS One 3: e2479.

Shurman B, Horan WP, Nuechterlein KH (2005). Schizophrenia patients demonstrate a distinctive pattern of decision-making impairment on the Iowa Gambling Task. Schizophr Res 72: 215-224.

Simon NW, Montgomery KS, Beas BS, Mitchell MR, LaSarge CL, Mendez IA et al (2011). Dopaminergic modulation of risky decision-making. J Neurosci 31: 17460-17470.

Spivak B, Mester R, Wittenberg N, Maman Z, Weizman A (1997). Reduction of aggressiveness and impulsiveness during clozapine treatment in chronic neuroleptic-resistant schizophrenic patients. Clin Neuropharmacol 20: 442-446.

St Onge JR, Floresco SB (2009). Dopaminergic modulation of riskbased decision making. Neuropsychopharmacol 34: 681-697.

Stanford MS, Mathias CW, Dougherty DM, Lake SL, Anderson NE, Patton JH (2009). Fifty years of the Barratt Impulsiveness Scale: an update and review. Personal Indiv Diff 47: 385-395.

Swann AC, Pazzaglia P, Nicholls A, Dougherty DM, Moeller FG (2003). Impulsivity and phase of illness in bipolar disorder. $J$ Affect Disord 73: 105-111.

van der Meer L, de Vos AE, Stiekema APM, Pijnenborg GHM, van Tol MJ, Nolen WA et al (2012). Insight in schizophrenia: involvement of self-reflection networks? Schizophr Bull 27: 27.

Ventura J, Green MF, Shaner A, Liberman RP (1993). Training and quality assurance with the Brief Psychiatric Rating Scale: 'The drift busters'. Int J Methods Psychiatr Res 3: 221-244.

White TL, Lejuez CW, de Wit H (2008). Personality and gender differences in effects of d-amphetamine on risk taking. Exp Clin Psychopharmacol 16: 565-570.

Young RC, Biggs JT, Ziegler VE, Meyer DA (1978). 'A rating scale for mania: reliability, validity and sensitivity.' Br J Psychiatry 133: 429-435.

Yücel M, Brewer WJ, Harrison BJ, Fornito A, O'Keefe GJ, Olver J et al (2007). Anterior cingulate activation in antipsychotic-naïve first-episode schizophrenia. Acta Psychiatr Scand 115: 155-158.

Supplementary Information accompanies the paper on the Neuropsychopharmacology website (http://www.nature.com/npp) 